\title{
Infections After Kidney Transplantation: The Bug Bear Of Kidney Transplantation In Tropics
}

\author{
Gopal Basu*
}

Department of Nephrology, Christian Medical College, Vellore, India

\begin{abstract}
Infections are the bugbear of kidney transplantation in the tropics, being responsible for majority of the deaths. Despite the several challenges posed by infections in kidney transplant recipient in the tropics, various developments have resulted in a decline in the rate of infections as well as their consequences. This review aims to be a basic overview of the common infections in KTR with an attempt to provide a unique tropical country perspective.
\end{abstract}

Keywords: CMV, hepatitis B, hepatitis C, infection, kidney, post-transplant, renal transplantation, transplantation, tropical, tuberculosis, urinary tract infection.

\section{INTRODUCTION}

In the four-decade history of kidney transplantation (KT) in India, infections have been the nemesis. Infections account for $45-75 \%$ of mortality in kidney transplant recipients (KTRs) in the tropics, unlike $15-30 \%$ in the west. Post transplant infections are an independent risk factor for mortality (Fig. 1). Infections are a common cause of hospitalizations among KTRs. Comparatively, the art of transplant medicine in the tropics involves a finer balancing of the immunosuppression, and is considerably challenging in view of both economic constraints and increased prevalence of infections. Infections in kidney transplant recipients is a vast and dynamic field and this article aims to provide a basic overview tropical in context and readers are referred to excellent reviews in the reference section for detailed discussions.

\section{GENERAL CONSIDERATIONS}

Infections in KTR broadly follow predictable pattern or "timetable" of infections [1]. The time table of infections among recipients in India [2] differs from that in the west.

There are several challenges in the surveillance, detection and management of infections in KTRs.

- The complex web of interactions between several factors such as the choice of immunosuppression, the net state of immunosuppression, risk factors in the recipient, ambient infectivity and prevalence in the community, pre-transplant screening, transfusion practices, prior exposure, immunization status, pathogenic virulence of the microbe and prophylactic practices determine the occurrence of infections in kidney transplant recipient. It is not surprising, that

*Address correspondence to this author at the Department of Nephrology, Christian Medical College, Vellore - 632004, India;

Tel: 00914162282053; Fax: 00914162232035;

E-mails: drbasug@gmail.com,drbasug@yahoo.co.in the rates and consequences of infections vary between different countries, centers and patients.

- Changes in immunosuppressants have changed the incidence, nature and consequences of infections in KTR. Hence, as newer immunosuppressive drugs and protocols emerge from the west, it is prudent to accept them carefully in the tropics with a close watch for infections. The risk of infection in a tropical country recipient is much higher than the population on which these drugs /protocols are initially studied

- The lack of clinically relevant immunological assays that measure either the immune function and degree of immunosuppression or the immune activity against specific pathogens, impairs our ability to titrate the immunosuppression accurately to prevent rejections without suffering infections.

- $\quad$ Early diagnosis of infections in immunosuppressed individuals is impaired by the blunting of inflammatory responses by immunosuppressants and altering the natural history of these infections. A severely immunosuppressed individual may not have febrile response to Tuberculosis (TB) or cytomegalovirus (CMV) disease till when it is severe $/$ disseminated.

- Infections may present closely resembling noninfectious complications. BK virus nephropathy present similar to graft rejection, while parvovirus B 19 infections presents as pure red cell aplasia.

- Infections in KTRs, especially in the tropics, do not follow "Occam's Razor". Multiple co-infections are a common feature, with immunosuppression and exposure being common underlying factors. It is well known for CMV disease to co-occur with recurrent Urinary Tract Infection (UTI) or TB. Hepatitis C virus $(\mathrm{HCV})$ infected patients suffer higher incidence of TB, CMV or Nocardia. Parasitic and fungal infections also may co-exist. 


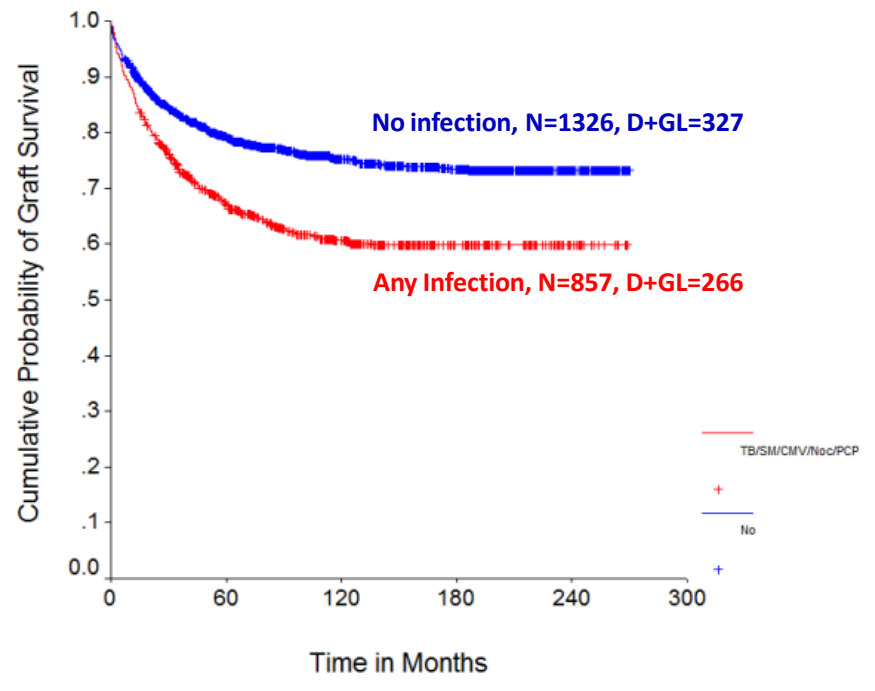

Fig. (1). Composite survival of patients with any of the following infections ( CMV, TB, Systemic Mycoses, Nocardiosis, PCP, Hepatitis B or C virus) among 2183 kidney transplant recipients at CMC Vellore between 1986-2007 (Courtesy Dr. George T John, Renal Medicine, Royal Brisbane and Womens hospital, Brisbane, Australia).

- The role of donor derived infections also assumes greater significance with the recent increase in the utilization of deceased donor organs in the tropics. There are several unique challenges in decision making and management in such situations.

- Antimicrobial therapy, often complex, has several interactions and drug toxicities in transplant recipients. For example, Rifampicin use has been proven to significantly impair both tacrolimus and mycophenolate levels, predisposing to rejection. Voriconazole use contraindicates sirolimus, and increases calcineurin inhibitor (CNI) levels multifold predisposing to CNI toxicity.

- $\quad$ The emergence of drug resistant microbes, unearthing of newer pathogens and re-emergence of certain pathogens (for eg. Pneumocystis jiroveci, M. leprae) have complicated the scenario. Most of the posttransplant urinary tract infections in many centers are due to extended spectrum beta-lactamase producing gram negative bacilli such as $E$. coli, requiring high end antibiotics such as carbapenems, which until recently were considered reserve drugs.

- The increasing number of transplants performed in HIV infected individuals have thrown open newer challenges in terms of drug interactions and novel infections.

Despite several challenges, the mortality due to infections in KTR has considerably improved in the last three decades. There are several important developments that have led to this decline in infective risk of our patients and a reduction in mortality due to infections in the tropics.

- General improvement in healthcare facilities, sanitation, availability of clean food and water are perceived as important factors, though difficult to unequivocally prove in the setting of kidney transplantation.

- Improved understanding of the pathogenesis-of, and the immune-response-to infections have helped develop better diagnostics, risk predictions and management strategies. Better reporting of infections and clinical outcomes add to the body of literature and consequently the understanding of infections.

- Early diagnosis with high index of suspicion is now made possible by several advances in diagnostics including imaging as well as molecular techniques. Such medical facilities are now more commonly available to patients in the tropics, than two decades ago. In addition, both patients and clinicians are more aware of the need for careful follow up of post transplant recipients.

- Therapeutic drug monitoring (TDM) along with assessment of immune function and immunological as well as infective risk stratification have resulted in personalized, tailored and judicious immunosuppression use in the tropics, avoiding both over- and under-immunosuppression.

- Development of specific and targeted immunosuppressive drugs has helped avoid reliance on general immunosuppressive strategies, thereby reducing risk of several infections.

- Vaccination has a significant effect on reducing incidence of infections in both the community and KTRs. This is evident with Hepatitis B infection.

- $\quad$ Effective antimicrobial prophylactic strategies such as the trimethoprim-sulfamethoxazole (TMP-SMZ) prophylaxis for Pneumocystis jirovecii pneumonia and Valganciclvir prophylaxis for CMV, have resulted in remarkable reduction in these specific infections.

- Improvement in therapeutics has simplified treatment of infections. Advent of oral valganciclovir therapy has reduced the complexity of treating CMV with parenteral ganciclovir.

- Adoption of effective infection control practices have helped in prevention of infections, consequently saving lives.

\section{SPECIFIC INFECTIONS}

\section{Urinary Tract Infections}

Urinary Tract Infections (UTI) are the most common bacterial infections and a common cause of sepsis after kidney transplantation. The classical risk factors for UTI include urological native kidney disease in the recipient, prolonged catheterization post kidney transplantation, improper catheterization practices, insertion of DJ stents and past history of recurrent urinary tract infections [3]. Escherichia coli is the most common uropathogen. Most UTI occur within first 3 months of kidney transplantation. Management usually involves sensitive antibiotic therapy for 2 weeks as for "complicated UTI" [4]. Graft pyelonephritis significantly affects graft survival in the tropics [5]. 
Treatment of asymptomatic bacteruria is controversial. Treatment of asymptomatic bacteruria beyond the first year of transplantation does not prevent symptomatic UTI [6]. The widespread occurrence of ESBL pathogen associated UTI is a cause for concern, and calls for effective and judicious antibiotic policies for control of emergence resistant organisms. Recurrent UTI in the absence of obvious urological / structural abnormality, especially with multiple drug resistant bacteria is a difficult clinical situation, and often managed with prolonged sensitive-antibiotic therapy ( 6 weeks) followed by continual cyclical prophylaxis. Complications such as emphysematous pyelonephritis, graft renal abscess need effective source control. Advances in culture techniques have helped in timely management of septic patients with sensitive antibiotics. Thus, potentially life threatening / graft threatening complications can effectively be managed with aggressive non-surgical measures [7].

Fungal UTI are often associated with prolonged catheterization, prolonged broad spectrum antibiotic use, DJ stent or presence of necrotic tissue (papillary necrosis / abscess). The common species of candida in the tropical countries is often resistant to fluconazole and therefore require treatment with amphotericin. Echinocandins do not concentrate in the urinary tract and are therefore less effective in treating candidal UTI [8]. If fluconazole (or any other triazole) is used, interaction with calcineurin inhibitors (CNI) and mTOR-inhibitors (mTORi) results in significant elevation of CNI / mTORi levels, necessitating dose reduction and careful monitoring of levels and renal function during and after therapy [9].

\section{Cytomegalovirus Disease}

CMV disease is the most common opportunistic viral infection among KTRs. It occurs in nearly $20-30 \%$ of patients not on prophylaxis [10] and the incidence reduced to $<10 \%$ among patients on Valganciclvoir prophylaxis [11]. Risk stratification based on donor and recipient sero-status has been advocated, but less useful in the tropics, in view of high prevalence of CMV seropositivity (CMV IgG), observed in $\sim 98 \%$ of the individuals in tropical countries [12], making the possibility of both $\mathrm{D}+/ \mathrm{R}$ - and D-/R- status a rare occurrence.

CMV infection refers to evidence of CMV replication without any symptom or organ involvement. CMV disease refers to replication of CMV virus with symptoms, classically manifesting either as "CMV Syndrome" - a flu like syndrome associated with leucopenia, thrombocytopenia or with specific organ involvement - involving gastrointestinal tract, liver, eye, lungs, etc. Apart from these direct effects of CMV, there are several potentially ominous indirect effects with immunomodulatory as well as inflammatory activity, such as Chronic allograft dysfunction, acute rejections, co-infections, accelerated $\mathrm{HCV}$ reactivation, new onset diabetes after transplantation, post-transplant lymphoproliferative diseases, cardiovascular disease, increased mortality and transplant vasculopathy (arterial stenosis or thrombosis) [11]. CMV disease, in the nonprophylaxis recipient occurs usually within the first three months, and poses a significant challenge to the physician trying to balance immunosuppression to recover from CMV at the same time prevent acute rejection at this early phase of transplant.

The risk factors for CMV disease include, donorrecipient serostatus (highest risk for $\mathrm{D}+/ \mathrm{R}$ - and lowest risk for D-/R-), immunosuppression with CNI, use of lymphocyte depleting antibody (induction/ anti-rejection therapy), acute rejection episodes, elderly age and co infection with $\mathrm{TB}$, $\mathrm{HCV}$, other viruses. However, use of prophylaxis, a pretransplant D-/R- serostatus, and use of mTORi are protective factors.

Diagnosis by CMV specific IgM results in many false positive and false negative results, and is not useful. CMV PP65 antigenemia by indirect immunoflourescnce in circulating leukocytes, though cumbersome, subjective and semiquantitative, has comparable sensitivity to PCR, and provides a reasonable alternative in pre-emptive monitoring as well as in guiding treatment response [13]. However, this test is limited by need to be performed within few hours of sampling and lesser utility in leukopenic patients. Nevertheless, at a fraction of the cost of the standard NATs, this is indeed a useful tool in resource poor settings. Quantitative nucleic acid testing (QPCR) assays are the goldstandard method for measuring viremia. Standardization of this technique and uniform reporting of results in IU/ml [14] across all centers is the need of the hour. At Christian Medical College (CMC) Vellore, a PCR count (interpreted after the sample is tested in duplicate) of $\geq 141$ genome copies/ml is considered positive, and a count of $\geq 1000$ genome copies $/ \mathrm{ml}$ to be clinically significant.

Higher CMV load in the early phase of transplant is associated with dissemination and multiple organ involvement. However, patients with late-onset CMV disease especially involving gastrointestinal tract or retina may paradoxically have few or no detectable copies of CMV genome in blood [15]. Thus tissue diagnosis by histopathology (or typical lesions in retinal examination) assumes significance. Owl-eyed nuclear inclusions are characteristic features of CMV cytopathology [16].

Prevention of CMV can be done either be with a preemptive therapy or a prophylaxis based strategy. Pre-emptive therapy involves regular periodic (ideally weekly) monitoring of CMV viremia, and responding to a rise in viremia beyond a pre-set threshold with antiviral therapy. Prophylaxis strategy involves either risk stratified or universal administration of prophylactic antiviral therapy for a predetermined duration [17]. Valganciclovir prophylaxis reduces the rate of CMV Disease from $25-30 \%$ to $5-10 \%$. Prophylaxis requires a daily dose of $900 \mathrm{mg}$ of valganciclovir once a day for normal renal function and 450 $\mathrm{mg}$ once daily for patients with eGFR between $40-59 \mathrm{ml} / \mathrm{min}$ [18]. Prophylaxis not only reduced the clinical CMV disease episodes, recurrence, or severity of the disease, but also indirect effects such as fewer opportunistic infections, improvement in graft and patient survival, reduction in PTLD, other viral infections. Compared to the pre-emptive strategy prophylaxis is logistically easier, with lesser monitoring cost, but higher cost of drug therapy [11]. Overall, the universal prophylaxis (especially for 200 days duration) was cost-effective when indirect benefits were also accounted for $[19,20]$. Problems with prophylactic strategy include frequent leucopenia, increase in late onset CMV 
disease, and emergence of resistant CMV. Leucopenia is often managed with reduction in antiproliferative immunosuppressant or temporary and complete withdrawal of Valganciclovir rather than dose reduction [21]. Prophylaxis in patients with D-/R- status or those at a higher risk of developing Hepres simplex or Varicella infections, could be done with Valacyclovir / Acyclovir. However, the doses of these drugs required for CMV prevention is considerably higher and potentially toxic [11].

In the tropics, despite predominantly serving $\mathrm{D}+/ \mathrm{R}+$ donor-recipient pairs, many centers perform kidney transplantation without induction agents and find the additional cost of valganciclovir prophylaxis untenable. The debate on which is the better preventive strategy has several considerations. In the tropics, universal prophylaxis has higher direct costs than pre-emptive testing monthly for 6 months (even with CMV Quantitative PCR). Use of cheaper and equally sensitive CMV PP65 testing is attractive and economical option. Nevertheless, the strength of the preemptive protocols lies in excellent follow up, regular and frequent monitoring with a robust, freely available test and uniform pre-defined cut-offs for intervention. Such logistics are not always feasible in the tropics. For example, access to reliable CMV testing is a problem in several smaller centers as well as a few big centers in the tropics. Patients in tropics may have to travel long distances to reach their transplant centers, making frequent visits inconvenient. In addition, introduction of low cost generics, liberal patent laws and a price control policy, the scenario is bound to change in favor of universal prophylaxis. The benefit of the several indirect effects as well as a "legacy effect" of prophylaxis compared to pre-emptive monitoring, especially in reduction of CMV recurrence rates seems attractive. Therefore the center may decide on either of these strategies based on the logistics and costs involved. However, as the costs of prophylaxis decline further, universal prophylaxis will be widely used for its simplicity, less "intensive" approach, and the "legacy effect". At CMC Vellore, where most transplant recipient receive basiliximab or Thymoglobulin induction, Valganciclovir prophylaxis is offered universally, and has resulted in a 50\% reduction in post transplant CMV disease, without significant increase in resistant CMV (Fig. 2) [22].

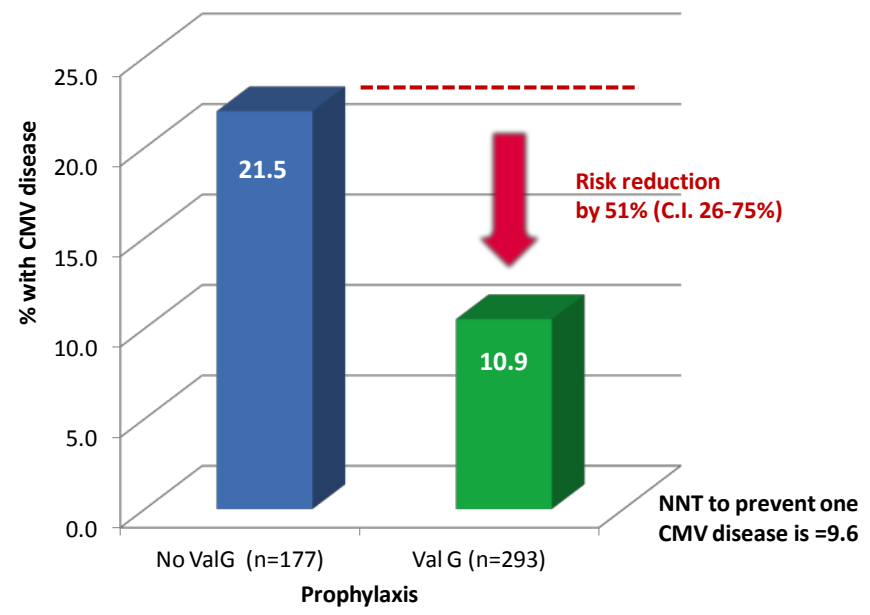

Fig. (2). Valganciclovir prophylaxis reduced CMV disease by $50 \%$ among 470 KTR in CMC Vellore studied between 2006 and 2011.
Treatment of CMV disease with antiviral agent consists of an initial induction phase followed by a maintenance phase of secondary prophylaxis [18]. Treatment of established CMV disease has undergone a sea change with the VICTOR study proving the non-inferiority of oral valganciclovir to IV ganciclvoir therapy in non-severe CMV disease [23]. Avoidance of central line insertion, admission for intiation of ganciclovir and the need for IV infusion has reduced the cost and logistic burden of treating CMV disease. In addition, cost of valganciclovir therapy in patients with reduced renal function is considerably less compared to IV ganciclovir, due to availability of only $250 \mathrm{mg}$ vials of ganciclovir of which only a fraction can be used in a day (due to renal dose adjusting), especially with a restriction that a reconstituted vial must be used within 48 hours. However, for severe CMV disease or retinal disease, IV ganciclovir is still the preferred agent [11]. Induction phase of therapy should be ideally continued for a minimum of 3 weeks or till when two consecutive assays of CMV are negative (a week apart) [11]. Failure to respond to appropriate therapy for $>3$ weeks should result in incremental dosing initially along with testing for resistance mutations (UL97, UL54) [21].

Emergence of resistant CMV is a rare but ominous problem. Testing for resistance mutations such as the UL97 or UL54 mutations, as well as therapeutic options such as foscarnet [21] are not widely available in tropics. Leflunomide may be useful in prophylactic agent as well as an adjunct for treatment of resistant CMV [24,25]. Other agents that may be used for resistant $\mathrm{CMV}$ include cidofvir, maribavir and artesunate [26].

\section{Mycobacterium Tuberculosis}

Tuberculosis (TB) is endemic in the tropics. KTRs (KTR) are at high risk of developing TB. The incidence of TB in KTR in tropics has been declining from a high of $15 \%$ to about $5 \%[27,28]$, a rate still higher than those in the developed nations. The risk factors for post transplant TB in tropical KTRs include higher age, diabetes mellitus (pre transplant), new onset diabetes after transplantation and presence of co-existing infections (systemic mycoses, CMV, $\mathrm{HCV}$, Pneumocystis jirovecii, Nocardia) indicating a higher net immunosuppressed state [29]. Use of Tacrolimus and Mycophenolate has also advanced the time of onset of tuberculosis after transplantation [30]. Novel risk factors such as vitamin D deficiency also contribute in the tropics [31].

Clinical TB in KTRs manifest differently from that in the general population, with increased prevalence of extrapulmonary / disseminated involvement in about 30-50\% [29]. Pulmonary involvement is the most common, followed by disseminated TB, GI involvement, and other extrapulmonary sites. $10-20 \%$ of patients present with pyrexia of unknown origin [29]. Organ involvement is similar to that seen in general population, though rare manifestations (such as intussusception) are possible [32].

The diagnosis of active TB in KTR often requires histological or microbiological proof of Mycobacterium tuberculosis (M.TB) invasion. In tropical endemic areas neither tuberculin skin testing (TST) and nor interferon 
gamma release assays (QuantiFERON TB Gold - QTB) are useful. Microbiological diagnosis by gastric juice AFB smear increases the yield in situations where AFB smear of sputum is negative in patients with pulmonary involvement [33]. The automated advanced culture techniques such as the MGIT system have reduced the waiting time for culture growth of M.TB from tissue/blood. Advances in imaging such as PET scan and BOLD MRI have helped in rapid localization and differentiation of TB in selected cases [34]. Effusive manifestations of TB can be diagnosed with fluid analysis. Adenosine deaminsae levels in pleural fluid add to the routine fluid analysis in increasing the probability of diagnosing tuberculosis [35]. The Polymerase chain reaction based nucleic acid testing for TB is sensitive and not only helps in rapid detection of M.TB in blood, body fluids and tissue samples (biopsy), but also screen for mutational resistance to anti-TB drugs (Rifampicin).

Latent tuberculosis [36] is endemic in the tropics, occurring in the vast majority of general population including prospective KTRs. Neither tuberculin skin testing (TST), nor interferon gamma release assays (QuantiFERON TB Gold) have been useful to diagnosing active tuberculosis in immunosuppressed individuals in the tropics [37,38]. In view of the widespread use of BCG vaccination and possible TB exposure from early childhood among tropical population, along with possible anergy to [38] to tuberculin antigen exposure in tropical CKD patients both TST and QTB have poor likelihood ratios for predicting latent as well as active TB.

Use of Isoniazid (INH) for primary prophylaxis in KTR is controversial. The benefit of INH prophylaxis in reducing incidence of post transplant TB in KTRs of TB - endemic region, over the risk of development of drug resistant $\mathrm{TB}$ with INH montherapy exposure is unclear. Despite proven protective efficacy in clinical trials in endemic countries such as India and Pakistan [39-41], INH primary prophylaxis is often not practiced due to fear of inducing primary INH resistance of Mycobacterium tuberculosis and the risk of INH hepatotoxicity. The incidence of drug resistance to INH and/or Rifampicin could be as high as $20 \%$ among Indian KTR with TB [42]. Neither TST nor QTB help in stratifying or selecting tropical patients to receive primary INH prophylaxis. In endemic tropical countries, anti-TB therapy is reserved for active manifest TB disease rather than the latent infection. However, in view of previous TB being a significant risk factor for reactivation in KTR even in the tropics, some centers, including CMC Vellore use INH as secondary prophylaxis among KTRs, after an episode of TB for a variable period of 2 years to life long.

There is no single optimum therapeutic strategy for TB in KTR. In the past few decades, there are few reports of successful treatment of Post transplant TB with the regular Rifampicin based four drug regimen for 6 months to 1 year [43]. Centers using Rifampicin based regimen, would usually treat patients for 9 to 12 months. Rifampicin induces hepatic microsomal enzyme systems thereby increasing the clearance of Calcineurin inhibitors (CNI) [44], mTOR inhibitors (mTORi) [45,46], steroids and Mycophenolate [47] reducing their bioavailability and efficacy, resulting in increased risk of acute rejection and graft loss [48]. The dose of immunosuppressants has to be adjusted with TDM to maintain their levels within therapeutic range. Reports of successful use of non-Rifampicin based anti-TB therapy although for an extended duration (18 months) exist [29,49]. Rifampicin sparing regimens have often used flouroquinolones (for e.g., Ofloxacin). At CMC Vellore, the protocol for anti TB therapy includes 3 months of pyrazinamide, 9-12 months of ofloxacin, 18 months of Ethambutol and INH along with B6. This is often followed by secondary INH prophylaxis for a prolonged and variable period. Rifabutin has similar drug interactions as Rifampicin but of a lesser degree. However, experience with use of Rifabutin [50-52] in the tropics is limited. An important concern is the increasing incidence of fluroquinolone resistance among M.TB isolates in tropics (non KTR). If an interacting drug such as Rifampicin is used, it is advisable to monitor all the affected drug levels, which should include CNI, mTORi as well as MPA. MPA monitoring is necessitated by the interaction of Rifampicin [47], reducing its bioavailability, considering the association of lower levels of MPA with acute rejection episodes in KTRs [53-55].

Reduction of immunosuppression is necessary, to avoid multiple co-infections in KTRs with TB. Assessment for Vitamin D deficiency and replenishing $25 \mathrm{OH}$ Vitamin D stores are prudent steps in the current management of active TB in KTR [56].

Immune reconstitution inflammatory syndrome (IRS) occurs in KTR with TB after initiating anti-TB therapy, along with reduction in immunosuppression. IRS manifests with worsening symptoms at the site of initial infection after 2-6 weeks of therapy. The risk factors include Rifampicin use and presence of CMV disease [57]. IRS, potentially life threatening, could be managed with continuation of anti-TB therapy and temporary increase in steroid dose to control the inflammation.

\section{BK Polyoma Virus Infection}

BK polyoma virus associated nephropathy affects 10 $15 \%$ of KTRs, resulting in graft loss in $10-80 \%$ of the affected individuals. The virus is urotropic, and often ubiquitous in the tropics. BK viruria precedes viremia $(30 \%)$ and viremia precedes nephropathy $(30-50 \%)$ by a median of $8-10$ weeks. BK virus associated nephropathy (BKVAN) occurs mostly within the first 3-6 months, commonly within the first 18 months and rarely after 2 years $(<5 \%)$ of transplant [58].

The risk factors for BK virus nephropathy include high net state of immunosuppression, older recipient age, male recipients, female donors, deceased donors, delayed graft function, HLA mismatch transplants, acute rejection episodes and its therapy [59]. BKVAN manifests with gradually progressive decline in renal function. Ureteric stricture and obstruction are also possible but rare manifestations of BK virus infection [58].

Diagnosis of BKVAN is by detection of virus in urine, blood and tissue. Renal histology of BKVAN is characterized by tubulointerstitial inflammation that is not easily differentiated from acute rejection [58]. Diagnosis is confirmed by BK virus quantitative PCR for viremia and a histological diagnosis made by presence of atypical cells with basophilic inclusions and /or SV40 immunostain 
positive tubular epithelial cells. Urine Decoy cells have been found to be specific but rarely sensitive enough to be recommended for routine screening. Presence of viral clusters called "Haufen" in urine electron microscopy is sensitive and specific but not a practical means for screening in tropical countries. Transplant centers in tropics do not have easy access to PCR testing for BK virus, especially the quantitative PCR. Routine screening for BK virus is recommended by Plasma NAT (BK PCR) performed at least monthly for the first 3-6 months after transplant, every 3 months until the end of the second year if an unexplained increase in serum creatinine occurs; and after treatment for acute rejection. Viremia of $4 \log$ genome copies are significantly associated with BKVAN but negative Urine PCR has a very high negative predictive value. Prevention of BKVAN in a viremic individual is primarily by reduction in immunosuppression. High positive BK Viremic recipients should undergo renal allograft biopsy, allowing for early detection and intervention and therefore a better prognosis [60].

Management of BK virus nephropathy is by reduction in immunosuppression. CNI targets have to be reduced to Tac trough levels $<4 \mathrm{ng} / \mathrm{ml}$, Cyclosporine levels $\sim 100 \mathrm{ng} / \mathrm{ml}$. Conversion of Tac to CsA may also be undertaken. Mycophenolate mofetil (MMF) is usually halved in dose, withdrawn or converted to Aza or mTORi [61]. Conversion of $\mathrm{CNI} / \mathrm{MMF}$ to mTORi is also recommended. Addition of drugs with antiviral properties is controversial though anecdotal evidence of beneficial effect of leflunomide exists [62]. At CMC Vellore, $60 \%$ of the recipients diagnosed to have BK viremia with graft dysfunction at a median of 18 months, demonstrated stabilization/improvement of graft function with immunosuppression reduction with addition of leflunomide (Fig. 3) [63]. Patients who have lost renal allografts to BK virus nephropathy may need graft nephrectomy and a non-viremic status before undergoing re-transplantation [64].

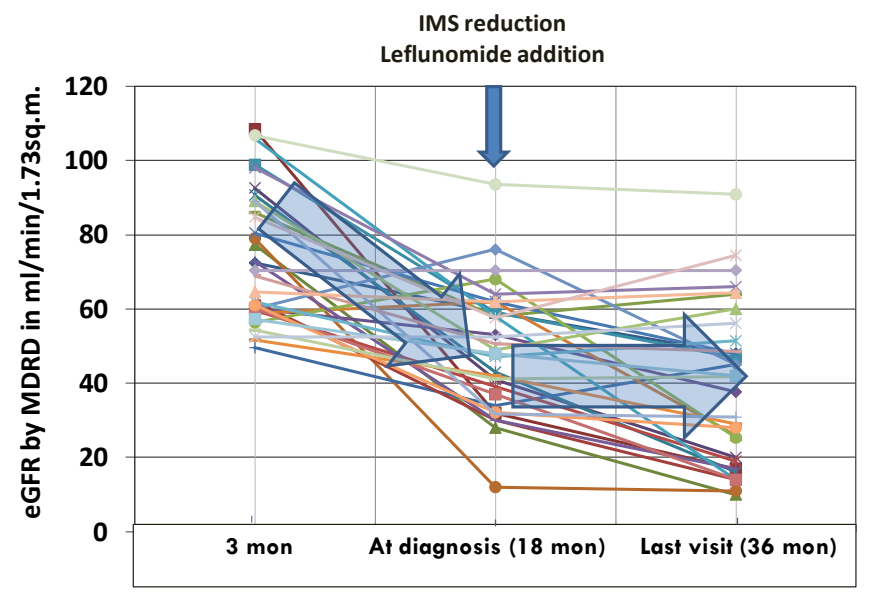

Fig. (3). The stabilization of eGFR among BKviremic KTRs at CMC Vellore on treatment with Leflunomide + Immunosuppression reduction - a study of $33 \mathrm{BK}$ viremic patients among $458 \mathrm{KTRs}$ studied between 2006 and 2011 values in pa.

\section{Herpes Simplex Virus Infection}

Herpes simplex virus infections (painful, grouped ulcerating vesicles in the perioral, genital regions) may occur frequently in KTRs either due to high net immunosuppressed state or during episodes of febrile illnesses [65]. Diagnosis is often by Tzanck smear and rarely requires blood PCR of HSV. HSV is usually treated with oral acyclovir in view of immunosuppressed state. Local application of acyclovir ointment may be undertaken as an additional measure. Patients with recurrent pre transplant HSV should receive Valacyclovir prophylaxis, especially if not on Valganiclvir prophylaxis and if it is a CMV D-/R- donor recipient pair. Disseminated disease or HSV meningitis is usually treated with IV acyclovir [65].

\section{Varicella Zoster Virus Infection}

Varicella presents as primary Chicken pox or as secondary reactivation - herpes zoster. $80-90 \%$ of children in the tropics have suffered chicken pox. VZV infections could be severe, disseminated and life threatening in immunosuppressed individuals. VZV infection in KTR is an indicator of degree of immunosuppression. Herpes zoster often precedes other opportunistic infections in KTRs, therefore an indication for reducing immunosuppression [66]. Patients with recurrent pre-transplant herpes zoster will need prophylaxis with Valacyclovir, if they are not receiving valganciclvoir. Treatment is with oral Valacyclovir / acyclovir for mild cases. Parenteral acyclovir therapy is reserved for disseminated / severe VZV infections [66]. Drug doses must be adjusted for renal function. Maintenance of hydration is important to prevent acyclovir induced AKI. Varicella zoster vaccine, a live attenuated vaccine is not advisable post transplant, but can be taken pretransplant, at least 4 weeks prior to immunosuppression [66].

\section{Epstein Barr Virus and Post Transplant Lympho- Proliferative Disease}

Epstein-Barr virus (EBV) infection in KTRs is associated with morbidity and mortality because of Post Transplant Lympho-proliferative Disease (PTLD). PTLD, results from spontaneous uncontrolled mono - or polymorphic proliferation of lymphoid cells, predominantly B cells and occasionally T cells [67]. $60-80 \%$ of PTLD is associated with EBV. An EBV D+/R- pair has a highest risk of PTLD. Other risk factors include, Lytic induction agent use, CNI use, prolonged immunosuppression, CMV disease, younger recipient, and recently, use of Belatacept [68]. EBV disease may also present with flu-like syndrome, lympadenopathy, or organ involvement such as hepatosplenomgealy, hepatitis, GI involvement, pneumonitis, leukopenia and occasionally hemophagocytic syndrome. EBV disease occurs early in D+/R- recipients, often within first 3-6 months. In the tropics with considerably higher ambient sero-prevalence, most patients do not suffer an EBV syndrome, and manifest PTLD much later into their transplant [69]. PTLD is diagnosed by biopsy along with immunohistochemistry and cytogenetics, and staged based on site, cell type, clonality and spread. Mortality can be $50-80 \%[69,70]$. Therapy often includes, reduction of immunosuppression (often prednisolone alone is continued with or without an mTORi), chemotherapy with Rituximab (monoclonal anti-CD20-Ab use) in B cell PTLDs, resulting in a $50-80 \%$ remission [71]. PTLD of the allograft may need surgical resection of the graft kidney. Survival 
varies from $35-60 \%$ depending on the stage. Though KDIGO guidelines propose monitoring EBV viral load by NAT in the first post-transplant week, then monthly for the first 3-6 months, then every 3 months until the end of first posttransplant year and after acute rejection therapy in high-risk groups [61], the test is often unavailable in many centers in the tropics and cost constraints prevent such intense monitoring. EBV PCR monitoring can also be used as a surrogate indicator of degree of immunosuppression, high viral loads prompting immunosuppression reduction and close follow up [61].

\section{Systemic Mycosis}

Systemic fungal infections affect $<5 \%$ of KTRs, and are often indicators of degree of immunosuppression [72]. Systemic mycoses are characterized by high mortality / graft loss especially in the tropics [73]. Apart from oropharyngoesophageal candidiasis and urinary tract infections, post transplant fungal infections often present as pneumonitis, meningitis or localized abscesses [74]. Risk factors for fungal infections include high dose steroid exposure, higher immunosuppression [53], multiple rejection episodes, poor graft function, elderly age, lytic induction agent use and coinfections with hepatitis C, CMV and TB [73,75]. Diagnosis is often by invasive sampling of fluid, or tissue from which the fungi are isolated and identified by microscopy and culture. Non-candidal-fungi (cryptococcus, histoplasma, fusarium, etc) are difficult to isolate and grow in culture or exhibit slower growth and delayed sporulation, thereby delaying speciation and specific diagnosis [76]. Speciation can now be done with advanced molecular techniques, but they are not widely available. Other additional tests such as galatomannan assay for aspergillus or latex agglutination for cryptococcus help in diagnosis. Therapy often is initiated empirically on high index of suspicion, especially in cases of pneumonitis. Choice of antifungal therapy should be determined by the expected fungus and the site of infection. Broad spectrum antifungal agents such as liposomal amphotericin have remained the agents of first choice for empirical therapy in severe infections [73]. Antifungal therapy with triazoles such as fluconazole, itraconazole or voriconazole results in inhibition of the hepatic microsomal CYP3A4 enzyme systems and consequent increase in the levels of CNI and mTORi, necessitating dose reduction and careful TDM during and after therapy. Voriconazole and mTORi should not be concurrently administered. There is very limited data to support routine antifungal prophylaxis in KTRs. KDIGO guidelines recommend oral and esophageal candidal prophylaxis with oral clotrimazole lozenges, nystatin, or fluconazole for 1-3 months after transplantation or a month after antilymphocyte antibody therapy [61].

\section{Pneumocystis jirovecii Pneumonia (PCP)}

Pneumocystis jirovecii pneumonia (PCP) is a life threatening opportunistic pulmonary infection in KTRs, contributing to significant morbidity and mortality. With universal TMP-SMZ prophylaxis for 6- 12 months the rate of PCP in KTR has reduced to about 0.8 cases $/ 1000$ patients after completion of one year of prophylaxis [77]. PCP in KTR is associated with $30-50 \%$ mortality. PCP, often seen after cessation of prophylaxis, typically manifests with fever, cough, dyspnea and characteristic hypoxia which is out of proportion to physical and radiologic findings. Diagnosis of PCP is usually by demonstration of the organism in sputum / bronchoalveolar lavage or in transbronchial lung biopsy tissue by indirect Immunoflourescence [78]. Computed tomography defines the extent of disease.

Trimethoprim-sulfamethoxazole (TMP-SMZ) is the agent of choice for prophylaxis [79] and have reduced PCP associated mortality significantly [80]. In addition, it prevents bacterial UTI, toxoplasmosis, cryptosporidiosis and nocardiosis. Daily TMP-SMZ prophylaxis is recommended in KTRs. Currently most KTRs in tropics receive TMP-SMZ (80mgTMP/day) prophylaxis for the initial six months. Patients intolerant to TMP-SMZ (Allergy or neutropenia) can be given dapsone $(100 \mathrm{mg} /$ day $)$, aerosolized pentamidine (300mg inhaled every 3-4 weeks - not available in India), atovaquone $(1500 \mathrm{mg} /$ day $)$ or the combination of clindamycin and pyrimethamine. Break through PCP infection while on prophylaxis is rare.

There is often a clustering of infections in KTRs [81-83]. CDC recommends isolation of patients with PCP during their hospital admission. There is growing evidence of late onset PCP occurring in patients who have received initial prophylaxis for 6 or 12 months [77,82,84]. Risk factors of such late onset PCP include treatment of acute rejection episodes, declining renal function as well as CMV disease $[82,85]$. The recent outbreak of late onset PCP in Australian KTRs has prompted a change in protocol, to continue TMPSMZ prophylaxis indefinitely in all their KTRs. In view of these risk factors patients who undergo treatment for acute rejection and CMV Disease should receive prophylaxis for the period of risk.

The disease should be treated with high dose TMP-SMZ $(15 \mathrm{mg} / \mathrm{Kg} / \mathrm{D}$ of TMP) for a period of 21 days, along with corticosteroids $(50 \mathrm{mg}$ twice daily for 7 days followed by tapering dose over next 2 weeks), especially if patient is hypoxic [86]. Alternatively inhaled pentamidine or clindamycin - pyrimethamine may be used.

\section{Hepatitis C Virus Infection}

Infection with Hepatitis $\mathrm{C}$ is an important problem, with alarming increase in prevalence among prospective recipient on hemodialysis in the tropics. Lack of adherence to universal precautions and good hospital infection control practices in dialysis centers in the tropics have increased the incidence of $\mathrm{HCV}$ infections in dialysis patients to epidemic proportions. Recognition of $\mathrm{HCV}$ infection before kidney and after transplantation is important. The first report from India came from CMC Vellore in 1992, when we tested for $\mathrm{HCV}$ antibodies using first and second-generation enzymelinked immunosorbent assay [87]. Currently, screening necessitates nucleic acid testing, since serology has poor sensitivity $[88,89]$, in view of poor antibody response. In addition, HCV infection may not be associated with abnormal liver function test results. At CMC Vellore, pre transplant $\mathrm{HCV}$ positive had reduced from $8 \%$ to $4 \%$ over the last decade [89]. HCV infected KTRs have poor survival and several direct consequences such as cryoglobulinemic glomerulonephritis or membranoproliferative GN or 
membranous nephropathy, accelerated progression to cirrhosis with fibrosing cholestatic hepatitis (FCH) and hepatocellular carcinoma and indirect complications such as new onset diabetes after transplantation, increased mortality, co-infections with CMV, TB, Nocardia and fungal infections [90], irrespective of the timing of infection. All HCVpositive potential recipients should undergo liver biopsy to know the degree of fibrosis / inflammation for risk assessment and decision making on treatment as well as prognosis after transplant. HCV Viremia increases and reactivation occurs in $2-10 \%$ of patients after transplantation [89]. Many KTRs with HCV infection have abnormal histology despite normal liver enzyme profiles. About 5-20\% of patients may develop cirrhosis within 3-7 years of follow up. Therefore monitoring is crucial with NATs as other noninvasive tests are not reliable. Fibroscan assessment of liver fibrosis may help offset the need for serial liver biopsies in this setting. Serial monitoring of viral load by PCR is necessary. While most patients progressed or retained the liver fibrosis, about $20 \%$ of patients showed a mild degree of improvement in the fibrosis after kidney transplantation. Pretransplant $\mathrm{HCV}$ infected recipients should be treated for $\mathrm{HCV}$ infection based on the liver biopsy findings. Response to interferon may vary while treating with Pegylated interferon and Ribvarin between 20$80 \%$ in various studies [91]. Addition of Ribavarin results in severe anemia, requiring large doses of Erythropoietin and increase transfusion requirements [92]. Pre transplant IFN therapy is associated with other benefits such as reduction in de novo HCV associated GN [93], lesser incidence of new onset diabetes after transplantation [94] and lesser rates of chronic allograft dysfunction [95].

Interferon therapy is associated with increased risk of rejection, making treatment of $\mathrm{HCV}$ infection in the post transplant setting difficult. Interferon therapy is undertaken only if the benefits outweigh the risk for rejection / graft dysfunction. Life threatening disease such as $\mathrm{FCH}$, may warrant emergency use of interferon and careful withdrawal of immunosuppression. Intensive immunosuppression use (such as ATG and monclonal lymphocytolytic agents) has been associated with increased risk of development of chronic liver disease among HCV infected KTRs. In the post transplant period, it is prudent to carefully evaluate and reduce immunosuppression, with close monitoring. The therapy of HCV infection post transplant is undergoing a sea change with the use of direct acting antiviral drugs such as Sofosbuvir and Ledipasvir, bot of which are safe and effective, and superior to interferon therapy.

\section{Hepatitis B Virus Infection}

The prevalence of $\mathrm{HBV}$ infection in CKD patients has been on the decline with effective and early vaccination strategies. At our center, the pre-transplant HBV prevalence has declined from $24 \%$ in 1980 's [96], to $12 \%$ in 1998 [72] to about $4 \%$ in 2008 [89]. Intramuscular double dose vaccination (at 0,1,2 and 6 months) should be initiated in the pre-dialysis CKD stages for better seroconversion [97]. Intradermal vaccination improves sero-conversion rates in those who fail the intramuscular protocol [98]. Diagnosis pre- and post-transplant requires HBV DNA PCR along with surface antigen and core antibody testing. About $10-20 \%$ of the Anti HBC Ab positive individuals are HBV DNA PCR positive [89]. Pre-transplant evaluation should also include Liver biopsy to assess for acute as well as chronic changes and to rule out cirrhosis. Pre-transplant antiviral therapy is widely used. Transplant is best timed after virologic remission. HBV infection is an independent risk factor for both mortality and graft loss [99]. In the absence of antiviral therapy, histologic progression to CLD was observed in $85 \%$ of individuals over 5 years [100]. Post transplant management remains unclear. Interferon therapy is not used post transplant, fearing rejection [61]. Rapid emergence of resistance with Lamivudine use has shifted the paradigm to use of Entecavir as a first line therapy, which has the lowest rate of drug resistance at $1 \%$ in 5 years. Adefovir and tenofovir may be second line agents but may cause nephrotoxicity with tubular dysfunction, tubular necrosis and occasional interstitial nephritis. Antiviral therapy is initiated preferably before or at least at the time of transplantation to prevent viral reactivation and progression of liver fibrosis. Post transplant, it is imperative to maintain the lowest possible steroid dose and minimize all immunosuppressive medications [61]. Post transplant monitoring should be performed with HBV quantitative PCR, liver enzymes, alpha fetoprotein, USG liver (screening for cirrhosis and hepatocellular carcinoma).

\section{Human Immunodeficiency Virus Infection}

HIV infection is no more a contraindication to kidney transplantation. Successful transplantation in HIV positive individuals have been reported from the west [101] as well as the tropics [102]. However, this development brings new challenges in management. The survival rates of HIV positive KTRs is similar to that of KTRs of age $>65$ years [103]. Criteria for eligibility includes well controlled HIV infection with CD4 counts $>200$ cells $/ \mathrm{ml}$, undetectable HIV RNA viral loads, and the absence of untreatable infections or malignancies. Challenges in management include increased rejection rate, pharmacokinetic interactions between antiretroviral agents (ART) and immunosuppressants, and complications related to hepatitis virus co-infection. Protease inhibitors are usually not used in such patients, to avoid drug interactions. In view of profound CD4 suppression [105], Thymoglobulin is reserved for the most immunologic high risk HIV recipients [104]. However, IL2 blockers such as basiliximab, tacrolimus and MMF are the preferred immunosuppressive drugs in HIV infected KTRs [106].

\section{Other Infections}

Nocardiosis: Nocardiosis presents as single or multiple abscesses and is associated with CNI use and co-existing chronic liver disease. Nocardiosis is often a marker of profound immunosuppression and susceptibility to tuberculosis, CMV. It has a significant effect on morbidity and mortality of KTRs in tropics [72]. Treatment involves surgical drainage of the pus along with combination antimicrobial therapy with TMP-SMZ along with one of the following: Imipenem, ciprofloxacin, clarithromycin, linezolid, minocycline, amoxicillin-clavulanate or Ceftriaxone (meropenem is less active than imipenem 
against Nocardia). Minocycline is a popular alternative to TMP-SMZ among allergic KTRs [107].

Kaposi Sarcoma: Human herpesvirus 8 infection in KTR results in Kaposi sarcoma, which manifests as mucocutaneous violaceous lesions, characterized histologically by sheets of fascicles of spindle cells seen with intervening slit like vascular spaces. Histopathological diagnosis could be aided by a positive HHV8 PCR in tissue / blood. Treatment involves reduction in immunosuppression and conversion to an mTORi based regime [108]. Local excision and systemic chemotherapy is rarely required. Resistant cases may benefit from adjunct therapy with drugs of anti-viral and anti-proliferative property such as Leflunomide [109] or foscarnet [110].

Parvovirus B 19: Parvovirus infection typically presents with severe anemia or pure red cell aplasia and rarely with hemophagocytic syndrome. Diagnosis is established by bone marrow examination demonstrating typical viral inclusions and serological testing of parvovirus B 19 IgM antibodies supported by quantitative PCR of PVB19 genome copies in blood [111]. Management in KTR is different from PRCA due to autoimmune etiology, and involves reduction of T cell immunosuppression (CNI) and administration of IVIG (400mg/Kg/Day for 5 days) [112].

Visceral Leishmaniasis: Visceral Leishmaniasis (Kala Azar) in kidney transplantation is common in the Indian subcontinent. Manifestations may be subtle in transplant recipients and diagnosis is often made by bone marrow or tissue biopsy (liver, spleen and occasionally the GI tract). Usual drug of choice is Liposomal amphotericin [113]. Miltefosine [114], though effective is often not widely available. Antimonials are rarely used in the present.

Dengue: The day biting Aedes egyptii mosquite borne dengue viral infection in KTRs could be life threatening especially if presenting with hemorrhagic manifestations or dengue shock syndrome [115]. Effective critical care with fluid resuscitation, blood transfusion support and conservative measures is the usual line of treatment.

Hansen's Disease: Mycobacterium leprae infection is not uncommon in the tropics and does occur in KTRs. Hansen's disease (with a reaction) should be one of the differential diagnosis in a patient with pyrexia of unknown origin, and evaluation by skin smears may be useful. KTR present with Hansen's disease, usually many years after transplantation. Management of reaction with prednisolone and thalidomide is essential and life saving. The usual MDT against Hansen's disease can be used. Rifampicin is better avoided in KTR for reasons discussed earlier.

Human Papilloma Virus: Human papilloma virus infection in KTR may manifest as viral warts or cervical dysplasia. Viral warts (verruca vulgaris) are often managed by local excision, reduction of immunosuppression, change of immunosuppression to mTORi. However a few cases respond only to alternative forms of therapy such as local application of Thuja occidentalis extract [116]. Cervical dysplasia, intraepithelial neoplasia is managed as per existing protocol for non KTR individuals. Human papilloma virus vaccine is not contraindicated, but its role in women KTR is unclear.

\section{Vaccination}

KDIGO recommends that KTRs should be given inactivated vaccines and live vaccines should be avoided in all but exceptional circumstances. In view of high dose immunosuppression in the first 6 months post-transplant, routine vaccination could be delayed for this period of time. While live attenuated vaccines such as Influenza, Varicella, Rotavirus, Measles, Mumps, Rubella, BCG can be administered in children and adults pre-transplant, they are not advisable to be taken after transplant. Inactivated polio vaccine is preferred over oral polio vaccine for the same reason. Most live attenuated vaccines, such as varicella vaccination should be administered at least 4 weeks prior to initiation of immunosuppression.

\section{CONCLUSION}

Infections are the bugbear of kidney transplant in tropics. The challenges they pose have been to a certain extent overcome with several interventions and advances. Improved understanding of immunosuppression, competing risks of rejection and infection, measured and tailored immunosuppression, advances in screening, diagnostic tests including imaging and molecular techniques, prophylactic intervention protocols and management strategies, have made it possible to manage and restrict the consequences of infections and work towards better patient survival among our KTRs.

\section{CONFLICT OF INTEREST}

The author confirms that this article content has no conflict of interest.

\section{ACKNOWLEDGEMENTS}

Declared none.

\section{REFERENCES}

[1] Fishman JA. Infection in solid-organ transplant recipients. N Engl J Med 2007; 357(25): 2601-14.

[2] John GT, Date A, Mathew CM, Jeyaseelan L, Jacob CK, Shastry JC. A time table for infections after renal transplantation in the tropics. Transplantation 1996; 61(6): 970-2.

[3] Lorenz EC, Cosio FG. The impact of urinary tract infections in renal transplant recipients. Kidney Int 2010; 78(8): 719-21.

[4] Parasuraman R, Julian K. Urinary tract infections in solid organ transplantation. Am J Transplant 2013; 13(Suppl 4): 327-36.

[5] Kamath NS, John GT, Neelakantan N, Kirubakaran MG, Jacob CK. Acute graft pyelonephritis following renal transplantation. Transpl Infect Dis 2006; 8(3): 140-7.

[6] Moradi M, Abbasi M, Moradi A, Boskabadi A, Jalali A. Effect of antibiotic therapy on asymptomatic bacteriuria in KTRs. Urol J 2005; 2(1): 32-5.

[7] Alexander S, Varughese S, David VG, et al. Extensive emphysematous pyelonephritis in a renal allograft treated conservatively: case report and review of the literature. Transpl Infect Dis 2012; 14(6): E150-5.

[8] Silveira FP, Kusne S. Candida infections in solid organ transplantation. Am J Transplant 2013; 13(Suppl 4): 220-7.

[9] Trofe-Clark J, Lemonovich TL. Interactions between anti-infective agents and immunosuppressants in solid organ transplantation. Am J Transplant 2013; 13(Suppl 4): 318-26.

[10] Rao M, Finny GJ, Abraham P, et al. Cytomegalovirus infection in a seroendemic renal transplant population: a longitudinal study of virological markers. Nephron 2000; 84(4): 367-73. 
[11] Kotton CN. CMV: Prevention, diagnosis and therapy. Am J Transplant 2013; 13(Suppl 3): 24-40; quiz

[12] Mukundan P, Jadhav M, John TJ. Prevalence of cytomegalovirus antibody in young children in Vellore. Indian J Med Res 1977; 65(5): 589-92.

[13] Razonable RR, Paya CV, Smith TF. Role of the laboratory in diagnosis and management of cytomegalovirus infection in hematopoietic stem cell and solid-organ transplant recipients. J Clin Microbiol 2002; 40(3): 746-52.

[14] Kraft CS, Armstrong WS, Caliendo AM. Interpreting quantitative cytomegalovirus DNA testing: understanding the laboratory perspective. Clin Infect Dis 2012; 54(12): 1793-7.

[15] Cummins NW, Deziel PJ, Abraham RS, Razonable RR. Deficiency of cytomegalovirus (CMV)-specific CD8+ $\mathrm{T}$ cells in patients presenting with late-onset CMV disease several years after transplantation. Transpl Infect Dis 2009; 11(1): 20-7.

[16] Mattes FM, McLaughlin JE, Emery VC, Clark DA, Griffiths PD. Histopathological detection of owl's eye inclusions is still specific for cytomegalovirus in the era of human herpesviruses 6 and 7 . J Clin Pathol 2000; 53(8): 612-4.

[17] Kotton CN, Hibberd PL. Travel medicine and transplant tourism in solid organ transplantation. Am J Transplant 2013; 13(Suppl 4): 337-47.

[18] Razonable RR, Humar A. Cytomegalovirus in solid organ transplantation. Am J Transplant 2013; 13(Suppl 4): 93-106.

[19] Blumberg EA, Hauser IA, Stanisic S, et al. Prolonged prophylaxis with valganciclovir is cost effective in reducing posttransplant cytomegalovirus disease within the United States. Transplantation. 2010; 90(12): 1420-6.

[20] Luan FL, Kommareddi M, Ojo AO. Universal prophylaxis is cost effective in cytomegalovirus serology-positive kidney transplant patients. Transplantation 2011; 91(2): 237-44.

[21] Kotton CN, Kumar D, Caliendo AM, et al. International consensus guidelines on the management of cytomegalovirus in solid organ transplantation. Transplantation 2010; 89(7): 779-95.

[22] Sudhakar G, Basu G, Valson AT, et al. Cytomegalovirus disease in KTRs in valganciclovir era. In: Ammanna VJ, Ed. $33^{\text {rd }}$ Annual Conference of the Indian Society of Nephrology Southern Chapter. Vijayawada, India 2013.

[23] Asberg A, Humar A, Rollag H, et al. Oral valganciclovir is noninferior to intravenous ganciclovir for the treatment of cytomegalovirus disease in solid organ transplant recipients. Am J Transplant 2007; 7(9): 2106-13.

[24] John GT, Manivannan J, Chandy S, et al. A prospective evaluation of leflunomide therapy for cytomegalovirus disease in renal transplant recipients. Transplant Proc 2005 ; 37(10): 4303-5.

[25] John GT, Manivannan J, Chandy S, Peter S, Jacob CK. Leflunomide therapy for cytomegalovirus disease in renal allograft recepients. Transplantation 2004; 77(9): 1460-1.

[26] Avery RK. Update in management of ganciclovir-resistant cytomegalovirus infection. Curr Opin Infect Dis 2008; 21(4): 4337 .

[27] Sundaram M, Adhikary SD, John GT, Kekre NS. Tuberculosis in renal transplant recipients. Indian J Urol 2008; 24(3): 396-400.

[28] Sakhuja V, Jha V, Varma PP, Joshi K, Chugh KS. The high incidence of tuberculosis among renal transplant recipients in India. Transplantation 1996; 61(2): 211-5.

[29] John GT, Shankar V, Abraham AM, Mukundan U, Thomas PP, Jacob CK. Risk factors for post-transplant tuberculosis. Kidney Int 2001; 60(3): 1148-53.

[30] Atasever A, Bacakoglu F, Toz H, et al. Tuberculosis in renal transplant recipients on various immunosuppressive regimens. Nephrol Dial Transplant 2005; 20(4): 797-802.

[31] Talat N, Perry S, Parsonnet J, Dawood G, Hussain R. Vitamin d deficiency and tuberculosis progression. Emerg Infect Dis 2010; 16(5): 853-5.

[32] Mohapatra A, Basu G, Sen I, et al. Tuberculosis in a renal allograft recipient presenting with intussusception. Indian J Nephrol 2012; 22(1): 52-6.

[33] John GT, Juneja R, Mukundan U, et al. Gastric aspiration for diagnosis of pulmonary tubercolosis in adult renal allograft recipients. Transplantation 1996; 61(6): 972-3.

[34] Koshy CG, Livingstone RS, Basu G, John GT. Renal allograft tuberculosis-an evaluation using blood oxygen level-dependent magnetic resonance imaging. Transplantation 2010; 89(1): 124-5.
[35] Chung JH, Kim YS, Kim SI, et al. The diagnostic value of the adenosine deaminase activity in the pleural fluid of renal transplant patients with tuberculous pleural effusion. Yonsei Med J 2004; 45(4): 661-4.

[36] Subramanian AK, Morris MI. Mycobacterium tuberculosis infections in solid organ transplantation. Am J Transplant 2013; 13 Suppl 4: 68-76.

[37] Agarwal SK, Gupta S, Bhowmik D, Mahajan S. Tuberculin skin test for the diagnosis of latent tuberculosis during renal replacement therapy in an endemic area: a single center study. Indian J Nephrol 2010; 20(3): 132-6.

[38] Shankar MS, Aravindan AN, Sohal PM, et al. The prevalence of tuberculin sensitivity and anergy in chronic renal failure in an endemic area: tuberculin test and the risk of post-transplant tuberculosis. Nephrol Dial Transplant 2005; 20(12): 2720-4.

[39] John GT, Thomas PP, Thomas M, et al. A double-blind randomized controlled trial of primary isoniazid prophylaxis in dialysis and transplant patients. Transplantation 1994; 57(11): 1683-4.

[40] Agarwal SK, Gupta S, Dash SC, Bhowmik D, Tiwari SC. Prospective randomised trial of isoniazid prophylaxis in renal transplant recipient. Int Urol Nephrol 2004; 36(3): 425-31.

[41] Naqvi R, Naqvi A, Akhtar S, et al. Use of isoniazid chemoprophylaxis in renal transplant recipients. Nephrol Dial Transplant 2010; 25(2): 634-7.

[42] John GT, Mukundan U, Vincent L, Jacob CK, Shastry JC. Primary drug resistance to Mycobacterium tuberculosis in renal transplant recipients. Natl Med J India 1995; 8(5): 211-2.

[43] al-Sulaiman MH, Dhar JM, al-Khader AA. Successful use of rifampicin in the treatment of tuberculosis in renal transplant patients immunosuppressed with cyclosporine. Transplantation 1990; 50(4): 597-8.

[44] Offermann G, Keller F, Molzahn M. Low cyclosporin A blood levels and acute graft rejection in a renal transplant recipient during rifampin treatment. Am J Nephrol 1985; 5(5): 385-7.

[45] Kovarik JM, Hartmann S, Figueiredo J, et al. Effect of rifampin on apparent clearance of everolimus. Ann Pharmacother 2002; 36(6): 981-5.

[46] Ngo BT, Pascoe M, Khan D. Drug interaction between rifampicin and sirolimus in transplant patients. Saudi J Kidney Dis Transpl 2011; 22(1): 112-5.

[47] Annapandian VM, Fleming DH, Mathew BS, John GT. Mycophenolic acid area under the curve recovery time following rifampicin withdrawal. Indian J Nephrol 2010; 20(1): 51-3.

[48] Singh N, Paterson DL. Mycobacterium tuberculosis infection in solid-organ transplant recipients: impact and implications for management. Clin Infect Dis 1998; 27(5): 1266-77.

[49] Vachharajani TJ, Oza UG, Phadke AG, Kirpalani AL. Tuberculosis in renal transplant recipients: rifampicin sparing treatment protocol. Int Urol Nephrol 2002; 34(4): 551-3.

[50] Lopez-Montes A, Gallego E, Lopez E, et al. Treatment of tuberculosis with rifabutin in a renal transplant recipient. Am J Kidney Dis 2004; 44(4): e59-63.

[51] Lee J, Yew WW, Wong CF, Wong PC, Chiu CS. Multidrugresistant tuberculosis in a lung transplant recipient. J Heart Lung Transplant 2003; 22(10): 1168-73.

[52] Vandevelde C, Chang A, Andrews D, Riggs W, Jewesson P. Rifampin and ansamycin interactions with cyclosporine after renal transplantation. Pharmacotherapy 1991; 11(1): 88-9.

[53] Basu G, Annapandian VM, Matthew BS, et al. Concentration controlled mycophenolate dosing in renal transplantation in India: 1283. Transplantation 2010; 90: 255.

[54] Le Meur Y, Buchler M, Thierry A, et al. Individualized mycophenolate mofetil dosing based on drug exposure significantly improves patient outcomes after renal transplantation. Am J Transplant 2007; 7(11): 2496-503.

[55] van Gelder T, Silva HT, de Fijter JW, et al. Comparing mycophenolate mofetil regimens for de novo renal transplant recipients: the fixed-dose concentration-controlled trial. Transplantation 2008; 86(8): 1043-51.

[56] Salahuddin N, Ali F, Hasan Z, et al. Vitamin D accelerates clinical recovery from tuberculosis: results of the SUCCINCT Study [Supplementary Cholecalciferol in recovery from tuberculosis]. A randomized, placebo-controlled, clinical trial of vitamin D supplementation in patients with pulmonary tuberculosis'. BMC Infect Dis 2013; 13: 22. 
[57] Sun HY, Munoz P, Torre-Cisneros J, et al. Mycobacterium tuberculosis-associated immune reconstitution syndrome in solidorgan transplant recipients. Transplantation 2013; 95(9): 1173-81.

[58] Hariharan S. BK virus nephritis after renal transplantation. Kidney Int 2006; 69(4): 655-62.

[59] Hirsch HH, Randhawa P. BK polyomavirus in solid organ transplantation. Am J Transplant 2013; 13(Suppl 4): 179-88.

[60] Buehrig CK, Lager DJ, Stegall MD, et al. Influence of surveillance renal allograft biopsy on diagnosis and prognosis of polyomavirusassociated nephropathy. Kidney Int 2003; 64(2): 665-73.

[61] KDIGO clinical practice guideline for the care of KTRs. Am J Transplant 2009; 9(Suppl 3): S1-155.

[62] Josephson MA, Gillen D, Javaid B, et al. Treatment of renal allograft polyoma BK virus infection with leflunomide. Transplantation 2006; 81(5): 704-10.

[63] Saravanan M, Basu G, Kodgire SV, et al. BK Viremia in renal allograft recipients. Indian J Transplant 2012; 6(4): 114-5.

[64] Geetha D, Sozio SM, Ghanta M, et al. Results of repeat renal transplantation after graft loss from BK virus nephropathy. Transplantation 2011; 92(7): 781-6.

[65] Wilck MB, Zuckerman RA. Herpes simplex virus in solid organ transplantation. Am J Transplant 2013; 13(Suppl 4): 121-7.

[66] Pergam SA, Limaye AP. Varicella zoster virus in solid organ transplantation. Am J Transplant 2013; 13(Suppl 4): 138-46.

[67] Mohapatra A, Viswabandya A, Samuel R, et al. NK/T-cell lymphoma in a renal transplant recipient and review of literature. Indian J Nephrol 2011; 21(1): 44-7.

[68] Allen UD, Preiksaitis JK. Epstein-Barr virus and posttransplant lymphoproliferative disorder in solid organ transplantation. Am J Transplant 2013; 13(Suppl 4): 107-20.

[69] Madhivanan S, John GT, Rajasekar T, et al. Lymphoproliferative disorders in renal transplant recipients: a single-centre experience. Natl Med J India 2006; 19(1): 50-1.

[70] Opelz G, Dohler B. Lymphomas after solid organ transplantation: a collaborative transplant study report. Am J Transplant 2004; 4(2): 222-30.

[71] Elstrom RL, Andreadis C, Aqui NA, et al. Treatment of PTLD with rituximab or chemotherapy. Am J Transplant 2006; 6(3): 569-76.

[72] John GT. Infections after renal transplantation in India. J Nephrol Renal Transplant [Review]. 2009; 2(1): 71-88.

[73] Tharayil John G, Shankar V, Talaulikar G, et al. Epidemiology of systemic mycoses among renal-transplant recipients in India. Transplantation 2003; 75(9): 1544-51.

[74] John GT, Mathew M, Snehalatha E, et al. Cryptococcosis in renal allograft recipients. Transplantation 1994; 58(7): 855-6.

[75] Osawa R, Alexander BD, Lortholary O, et al. Identifying predictors of central nervous system disease in solid organ transplant recipients with cryptococcosis. Transplantation 2012; 89(1): 69-74.

[76] Singh N, Lortholary O, Dromer F, et al. Central nervous system cryptococcosis in solid organ transplant recipients: clinical relevance of abnormal neuroimaging findings. Transplantation 2008; 86(5): 647-51.

[77] Gordon SM, LaRosa SP, Kalmadi S, et al. Should prophylaxis for Pneumocystis carinii pneumonia in solid organ transplant recipients ever be discontinued? Clin Infect Dis 1999; 28(2): 240-6.

[78] Martin SI, Fishman JA. Pneumocystis pneumonia in solid organ transplantation. Am J Transplant 2013; 13(Suppl 4): 272-9.

[79] Fishman JA. Prevention of infection caused by Pneumocystis carinii in transplant recipients. Clin Infect Dis 2001; 33(8): 1397405 .

[80] Fox BC, Sollinger HW, Belzer FO, Maki DG. A prospective, randomized, double-blind study of trimethoprim-sulfamethoxazole for prophylaxis of infection in renal transplantation: clinical efficacy, absorption of trimethoprim-sulfamethoxazole, effects on the microflora, and the cost-benefit of prophylaxis. Am J Med 1990; 89(3): 255-74.

[81] de Boer MG, de Fijter JW, Kroon FP. OuTBreaks and clustering of Pneumocystis pneumonia in KTRs: a systematic review. Med Mycol 2011; 49(7): 673-80.

[82] Eitner F, Hauser IA, Rettkowski O, et al. Risk factors for Pneumocystis jiroveci pneumonia $(\mathrm{PcP})$ in renal transplant recipients. Nephrol Dial Transplant 2011; 26(6): 2013-7.

[83] Arichi N, Kishikawa H, Mitsui Y, et al. Cluster ouTBreak of Pneumocystis pneumonia among kidney transplant patients within a single center. Transplant Proc 2009; 41(1): 170-2.
[84] Wang EH, Partovi N, Levy RD, et al. Pneumocystis pneumonia in solid organ transplant recipients: not yet an infection of the past. Transpl Infect Dis 2012; 14(5): 519-25.

[85] Arend SM, Westendorp RG, Kroon FP, et al. Rejection treatment and cytomegalovirus infection as risk factors for Pneumocystis carinii pneumonia in renal transplant recipients. Clin Infect Dis 1996; 22(6): 920-5.

[86] Pneumocystis jiroveci (formerly Pneumocystis carinii). Am J Transplant 2004; 4(Suppl 10): 135-41.

[87] Mathew CM, Jacob CK, John GT, et al. Hepatitis C virus infection in renal allograft recipients - a pilot study. Indian J Nephrol 1994; 4: 1 .

[88] Centers for Disease Control and Prevention (CDC). Hepatitis C virus transmission at an outpatient hemodialysis unit--New York, 2001-2008. MMWR Morb Mortal Wkly Rep 2009; 58(8): 189-94.

[89] Joseph R, Rebekah G, Basu G, et al. Hepatitis B and C profile in pre and post renal transplant patients - a single center study. Indian J Nephrol 2008; 18(Suppl): s50.

[90] John GT. Infections after renal transplantation in India. Transplant Rev (Orlando, Fla) 1999; 13(4): 183-91.

[91] Kim E, Ko HH, Yoshida EM. Treatment issues surrounding hepatitis $\mathrm{C}$ in renal transplantation: a review. Ann Hepatol 2011; 10(1): 5-14.

[92] Rendina M, Schena A, Castellaneta NM, et al. The treatment of chronic hepatitis $\mathrm{C}$ with peginterferon alfa-2a (40 $\mathrm{kDa})$ plus ribavirin in haemodialysed patients awaiting renal transplant. J Hepatol 2007; 46(5): 768-74.

[93] Cruzado JM, Casanovas-Taltavull T, Torras J, et al. Pretransplant interferon prevents hepatitis $\mathrm{C}$ virus-associated glomerulonephritis in renal allografts by HCV-RNA clearance. Am J Transplant 2003; 3(3): 357-60.

[94] Gursoy M, Guvener N, Koksal R, et al. Impact of HCV infection on development of posttransplantation diabetes mellitus in renal allograft recipients. Transplant Proc 2000; 32(3): 561-2.

[95] Mahmoud IM, Sobh MA, El-Habashi AF, et al. Interferon therapy in hemodialysis patients with chronic hepatitis $\mathrm{C}$ : study of tolerance, efficacy and post-transplantation course. Nephron Clin Pract 2005; 100(4): c133-9.

[96] Thomas P, Kirubakaran MG, Jacob CK, et al. Hepatitis B infection in a dialysis unit in South India. J Assoc Physic India 1987; 35(4): 284-5.

[97] George J, John GT, Jacob CK, Shastry JC. Active immunization against hepatitis $B$ infection of a haemodialysis population. Natl Med J India 1994; 7(3): 115-6.

[98] Vincent L, John GT, Abraham P, Jacob CK. An intradermal vaccine protocol against hepatitis $\mathrm{B}$ in a haemodialysis population. Natl Med J India 1998; 11(1): 48.

[99] Fabrizi F, Martin P, Dixit V, Kanwal F, Dulai G. HBsAg seropositive status and survival after renal transplantation: metaanalysis of observational studies. Am J Transplant 2005; 5(12): 2913-21.

[100] Fornairon S, Pol S, Legendre C, et al. The long-term virologic and pathologic impact of renal transplantation on chronic hepatitis $\mathrm{B}$ virus infection. Transplantation 1996; 62(2): 297-9.

[101] Stock PG, Roland ME, Carlson L, et al. Kidney and liver transplantation in human immunodeficiency virus-infected patients: a pilot safety and efficacy study. Transplantation 2003; 76(2): 3705 .

[102] Bansal SB, Singhal M, Ahlawat R, Kher V. Kidney transplantation in a patient with HIV disease. Indian J Nephrol 2009; 19(2): 77-9.

[103] Stock PG, Barin B, Murphy B, et al. Outcomes of kidney transplantation in HIV-infected recipients. N Engl J Med 2010; 363(21): 2004-14.

[104] Ciuffreda D, Pantaleo G, Pascual M. Effects of immunosuppressive drugs on HIV infection: implications for solid-organ transplantation. Transpl Int 2007; 20(8): 649-58.

[105] Carter JT, Melcher ML, Carlson LL, Roland ME, Stock PG. Thymoglobulin-associated $\mathrm{Cd} 4+\mathrm{T}$-cell depletion and infection risk in HIV-infected renal transplant recipients. Am J Transplant 2006; 6(4): 753-60.

[106] Trullas JC, Cofan F, Tuset M, et al. Renal transplantation in HIVinfected patients: 2010 update. Kidney Int 2011; 79(8): 825-42.

[107] Clark NM, Reid GE. Nocardia infections in solid organ transplantation. Am J Transplant 2013; 13(Suppl 4): 83-92. 
[108] Stallone G, Schena A, Infante B, et al. Sirolimus for Kaposi's sarcoma in renal-transplant recipients. N Engl J Med 2005; 352(13): 1317-23.

[109] Basu G, Mohapatra A, Manipadam MT, Mani SE, John GT. Leflunomide with low-dose everolimus for treatment of Kaposi's sarcoma in a renal allograft recipient. Nephrol Dial Transplant 2011; 26(10): 3412-5.

[110] Luppi M, Barozzi P, Rasini V, et al. Severe pancytopenia and hemophagocytosis after HHV-8 primary infection in a renal transplant patient successfully treated with foscarnet. Transplantation 2002; 74(1): 131-2.

[111] Eid AJ, Brown RA, Patel R, Razonable RR. Parvovirus B19 infection after transplantation: a review of 98 cases. Clin Infect Dis 2006; 43(1): 40-8.

[112] Liefeldt L, Buhl M, Schweickert B, et al. Eradication of parvovirus B19 infection after renal transplantation requires reduction of immunosuppression and high-dose immunoglobulin therapy. Nephrol Dial Transplant 2002; 17(10): 1840-2.

[113] Sundar S, Agrawal G, Rai M, Makharia MK, Murray HW. Treatment of Indian visceral leishmaniasis with single or daily infusions of low dose liposomal amphotericin B: randomised trial. BMJ 2001; 323(7310): 419-22.

[114] Sundar S, Jha TK, Thakur CP, et al. Oral miltefosine for Indian visceral leishmaniasis. N Engl J Med 2002; 347(22): 1739-46.

[115] Prasad N, Bhadauria D, Sharma RK, et al. Dengue virus infection in renal allograft recipients: a case series during 2010 ouTBreak. Transpl Infect Dis 2012; 14(2): 163-8.

[116] Joseph R, Pulimood SA, Abraham P, John GT. Successful treatment of verruca vulgaris with Thuja occidentalis in a renal allograft recipient. Indian J Nephrol 2013; 23(5): 362-4.

Received: August 10, 2014

Revised: August 10, 2014

Accepted: March 10, 2015

(C) Gopal Basu; Licensee Bentham Open .

This is an open access article licensed under the terms of the Creative Commons Attribution Non-Commercial License (http://creativecommons.org/licenses/by-nc/3.0/) which permits unrestricted, non-commercial use, distribution and reproduction in any medium, provided the work is properly cited. 